Article

\title{
Rapid Screening of Mentha spicata Essential Oil and L-Menthol in Mentha piperita Essential Oil by ATR-FTIR Spectroscopy Coupled with Multivariate Analyses
}

\author{
Osman Taylan ${ }^{1}{ }^{\oplus}$, Nur Cebi $^{2, *}$ and Osman Sagdic ${ }^{2}$ \\ 1 Department of Industrial Engineering, Faculty of Engineering, King Abdulaziz University, \\ Jeddah 21589, Saudi Arabia; otaylan@kau.edu.sa \\ 2 Department of Food Engineering, Faculty of Chemical and Metallurgical Engineering, \\ Yıldız Technical University, İstanbul 34210, Turkey; sagdic@gmail.com \\ * Correspondence: nurcebi@yildiz.edu.tr
}

Citation: Taylan, O.; Cebi, N.; Sagdic, O. Rapid Screening of Mentha spicata Essential Oil and L-Menthol in Mentha piperita Essential Oil by ATR-FTIR Spectroscopy Coupled with Multivariate Analyses. Foods 2021, 10, 202. https://doi.org/ 10.3390/foods10020202

Academic Editor:

Francesco Longobardi

Received: 31 December 2020

Accepted: 11 January 2021

Published: 20 January 2021

Publisher's Note: MDPI stays neutral with regard to jurisdictional claims in published maps and institutional affiliations.

Copyright: (c) 2021 by the authors. Licensee MDPI, Basel, Switzerland. This article is an open access article distributed under the terms and conditions of the Creative Commons Attribution (CC BY) license (https:/ / creativecommons.org/licenses/by/ $4.0 /$ )

\begin{abstract}
Mentha piperita essential oil (EO) has high economic importance because of its wide usage area and health-beneficial properties. Besides health-beneficial properties, Mentha piperita EO has great importance in the flavor and food industries because of its unique sensory and quality properties. High-valued essential oils are prone to being adulterated with economic motivations. This kind of adulteration deteriorates the quality of authentic essential oil, injures the consumers, and causes negative effects on the whole supply chain from producer to the consumer. The current research used fast, economic, robust, reliable, and effective ATR-FTIR spectroscopy coupled chemometrics of hierarchical cluster analysis(HCA), principal component analysis (PCA), partial least squares regression (PLSR) and principal component regression (PCR) for monitoring of Mentha spicata EO and L-menthol adulteration in Mentha piperita EOs. Adulterant contents (Mentha spicata and L-menthol) were successfully calculated using PLSR and PCR models. Standard error of the crossvalidation SECV values changed between 0.06 and 2.14. Additionally, bias and press values showed alteration between 0.06 and1.43 and 0.03 and 41.15, respectively. Authentic Mentha piperita was successfully distinguished from adulterated samples, Mentha spicata and L-menthol, by HCA and PCA analysis. The results showed that attenuated total reflectance-Fourier transform infrared (ATRFTIR) spectroscopy, coupled with chemometrics could be effectively used for monitoring various adulterants in essential oils.
\end{abstract}

Keywords: ATR-FTIR; Mentha piperita essential oil; PLSR; PCR; HCA; adulteration; chemometrics

\section{Introduction}

Mentha piperita belongs to the Lamiaceae family, is one of the most well-known and most utilized herbs throughout the world [1]. Mentha piperita is generally defined as peppermint, which is involved in numerous products manufactured in various industries such as flavor, fragrances, cosmetics, aromatherapy, and phytomedicine [2]. According to the international standard, Mentha piperita oil is defined as an essential oil which is obtained from the plant Mentha piperita by steam distillation of the aerial parts of the plant [3]. Mentha piperita EO has high economic importance because of its wide usage area and health-beneficial properties. A number of studies have reported that Mentha piperita, medicinal plant showed anti-inflammatory, analgesic, anti-fungal, antimicrobial, and central nervous system excitation effects [4].

Besides health-beneficial properties, Mentha piperita EO has great importance in the flavor and food industries because of its unique sensory and quality properties. Mentha piperita EO is used as an ingredient in various food and beverage products because it is announced as "generally recognized as safe" by food regulations. Researchers reported that Mentha piperita essential oil is used as a flavor agent in commercial products such 
as chewing gums, candies, chocolates, drinks, herbal tea preparations, cough drops, the tobacco industry, and more [5]. One can understand that Mentha piperita EO has high economic and industrial importance since it is involved in a wide variety of consumer products throughout the whole world.

Economically motivated adulteration occurs when fraudsters intentionally substitute an authentic product with cheaper or less valuable materials. The U.S. Food and Drug Administration (FDA) defined "economically motivated adulteration" as the fraudulent, intentional substitution or addition of a substance in a product for the purpose of increasing the apparent value of the product or reducing the cost of its production [6]. Economic importance and wide application area make Mentha piperita EO prone to economically motivated adulteration. In this context, the determination of the adulterants in the Mentha piperita EO is of great importance in order to maintain and ensure the quality of high-valued natural extract. Authenticity control of Mentha piperita EO may prevent dishonest trading, exploitation of consumers, deterioration of the authentic product, and food-safety related problems and health risks originated from adulterants.

According to the scientific reports, essentials oils are adulterated using cheaper oils, various diluents, and synthetic chemical flavor compounds [7]. Previous researches reported that frequently used adulterants of Mentha piperita EOs were synthetic menthol and essential oil of cheaper Mentha species [8]. These kinds of adulteration deteriorate the quality of authentic essential oil, injure the consumers, and causes negative effects on the whole supply chain from producer to the consumer [9]. There is a need for effective methodologies for discrimination of the authentic Mentha piperita EO from fraudulent samples or other Mentha species. Additionally, adulterants such as synthetic menthol and cheaper Mentha species should be detected and quantified by using strong analytical methodologies.

Quality standards of Mentha piperita EO were determined according to the international standard of "Oil of Pepermint (Mentha $\times$ piperita L.)". The quality of Mentha piperita $\mathrm{EO}$ was determined on the basis of various test parameters such as relative density, refractive index, optical rotation, miscibility in ethanol, acid value, and chromatographic profile [3]. These analyses are well-known and trustable, but they are time-consuming and may require toxic chemicals. There is a need for new, rapid, easy, robust, low-cost, eco-friendly analytical techniques for the determination of adulterants in Mentha piperita EO.

FTIR spectroscopy is known as a rapid, non-destructive, reliable, effective, and lowcost analytical technique that provides fingerprint information about the chemical structure of materials [10]. FTIR analyses could be performed using no or minimal sample preparation with very little amounts of essential oils. Previous researches reported the effectiveness of FTIR spectroscopy for quality control of essential oils [9]. The authenticity of natural extracts such as eucalyptus essential oil, lavandin essential oil, pure camellia oil, lavender oil, and geranium oil was successfully determined by using vibrational spectroscopy combined with chemometrics [11-15].

There are two primary aims of the current study: 1 . To quantify the adulterants Mentha spicata, EO and L-menthol in Mentha piperita EO, by using ATR-FTIR (attenuated total reflectance-Fourier transform infrared) spectroscopy coupled with multivariate analyses of PLSR (partial least squares regression) and PCR (principal component regression); 2. To distinguish authentic Mentha piperita EO from adulterated samples, Mentha spicata EO and L-menthol, using ATR-FTIR spectroscopy coupled with HCA (hierarchical cluster analysis) and PCA (principal component analysis)

\section{Materials and Methods}

\subsection{Devices}

Bruker Tensor 27 FTIR spectrometer (Bruker-Germany) was used for the collection of ATR-FTIR measurements. The spectral acquisition was performed in the spectral range of 400 to $4000 \mathrm{~cm}^{-1}$. ATR (attenuated total reflectance) unit was used in combination with 
FTIR spectrometer. Menhta piperita EO, Mentha spicata EO, and L-menthol were identified by using the library database (ATR-FTIR Complete Library) of FTIR spectrometer.

\subsection{Samples and Materials}

Authentic Mentha piperita EO $(n=3)$, Mentha spicata EO $(n=3)$ were obtained from the well-known flavor and fragrance companies in Turkey. L-menthol (99\%) was bought from a chemist's shop (Istanbul, Turkey).

\subsection{Essential Oils and Preparation of Spiked Samples}

Mentha piperita essential oils are presented as MP1, MP2, and MP3. Mentha spicata essential oils are presented as MS1, MS2, and MS3 in the figures and tables. Additionally, L-menthol is presented as LM1, LM2, LM3 in the figures and tables. Spiking of MP1, MP2, and MP3 with Mentha spicata and L-menthol was performed for the concentrations of 0,1 , $2,4,8,16,32,64$, and $100 \%(v / v)$. We used nine concentration levels to build calibration curves for each analyte. A total of 42 spiked samples were prepared in the scope of this research. Samples were kept at $4{ }^{\circ} \mathrm{C}$ in dark vials until spectral acquisition.

\subsection{ATR-FTIR Measurements}

The FTIR spectra of authentic and adulterated EO samples were recorded at the spectral range of 400 to $4000 \mathrm{~cm}^{-1} .20 \mu \mathrm{L}$ of data set samples were pipetted with the help of an automatic pipette $(20-200 \mu \mathrm{L})$. Samples $\left(25^{\circ} \mathrm{C}\right)$ were directly placed on the surface of the diamond ATR crystal. The pipette tip was changed for each different sample. Operation of FTIR spectrometer and data acquisition was accomplished using the software OPUS Version 7.2 (Bruker Gmbh). Spectra were subtracted against the background air spectrum. A total of 16 scans were accumulated for each sample with a spectral resolution of $4 \mathrm{~cm}^{-1}$. Measurements were repeated four times for each sample and the average spectra were obtained using the software OPUS Version 7.2.

\subsection{Multivariate Analyses}

\subsubsection{Discrimination of Authentic Mentha piperita Essential Oil}

Authentic Mentha piperita EOs were distinguished from adulterated samples, Mentha spicata and L-menthol by application of hierarchical cluster analysis (HCA) to the collected spectral data. The software OPUS Version 7.2 (Bruker, Germany) was employed for HCA. HCA was performed by selection of chemometrics parameters of Ward's algorithm and Euclidian distance. The spectral region of $4000-400 \mathrm{~cm}^{-1}$ was chosen to distinguish authentic Mentha piperita Essential Oil from spiked samples, Mentha spicata and L-menthol.

\subsubsection{Prediction of Mentha spicata and L-Menthol Contents of Mentha piperita Essential Oil}

Mentha spicata and L-Menthol contents of spiked samples were quantified utilizing PLSR (partial least squares regression) and PCR (principal component regression) multivariate analysis. Grams IQ (Galactic Industries Corp, Salem, N.H., USA) software was used to perform PLSR and PCR. Multivariate models (calibration and cross-validation) were built using nine spiking levels of $0,1,2,4,8,16,32,64$, and $100 \%(v / v)$. PLSR and PCR models were built on the basis of first and second degree derivatized FTIR spectra data. Different frequency regions were selected for each adulterant since the spectral range should include information describing the concentration variation of the analyte or other matrix constituents [16]. The current paper utilized frequency ranges of 1694 to $1651 \mathrm{~cm}^{-1}$ and 1066 to $1034 \mathrm{~cm}^{-1}$ for quantification of Mentha spicata EO and L-menthol, respectively.

\section{Results}

\subsection{Spectral Properties of Mentha piperita Essential Oil}

ATR-FTIR spectra of Mentha piperita EO, Mentha spicata EO and L-menthol are presented in Figure 1. ATR-FTIR spectrum of a substance presents the unique chemical composition which is specific to that substance [17]. The effectiveness and capabilities of 
FTIR spectroscopy provide opportunities to monitor adulterants and suspicious materials in food and beverage matrices. Previous contributions reported that quality control of highvalued natural extracts such as essential oils could be accomplished by using mid-infrared spectroscopy [9]. In the current research, three different Mentha piperita essential oils were included in the data set. All of them showed similar spectral characteristics. Prominent spectral bands were observed at 2953, 2921, 2870, 1709, 1454, 1369, 1246, 1045, 993, 976, 919,887 , and $844 \mathrm{~cm}^{-1}$. Table 1 presents the spectral ranges and related band assignments of essential oils. Previous studies reported that the spectral ranges of 3100 to $3000 \mathrm{~cm}^{-1}$ and 3150 to $3050 \mathrm{~cm}^{-1}$ include vibrations arising from the stretching vibrations of $\mathrm{C}-\mathrm{H}$ groups [18]. The band at $2953 \mathrm{~cm}^{-1}$ corresponds to the asymmetric stretching vibrations of $-\mathrm{C}-\mathrm{H},-\mathrm{CH}_{3}$, and $-\mathrm{CH}_{2}$ groups [19]. The peak at $2921 \mathrm{~cm}^{-1}$ and $2870 \mathrm{~cm}^{-1}$ could be assigned to the $-\mathrm{C}-\mathrm{H},-\mathrm{CH}_{2}$ asymmetric stretching and $-\mathrm{C}-\mathrm{H},-\mathrm{CH}$ symmetric stretching vibrations, respectively [19].

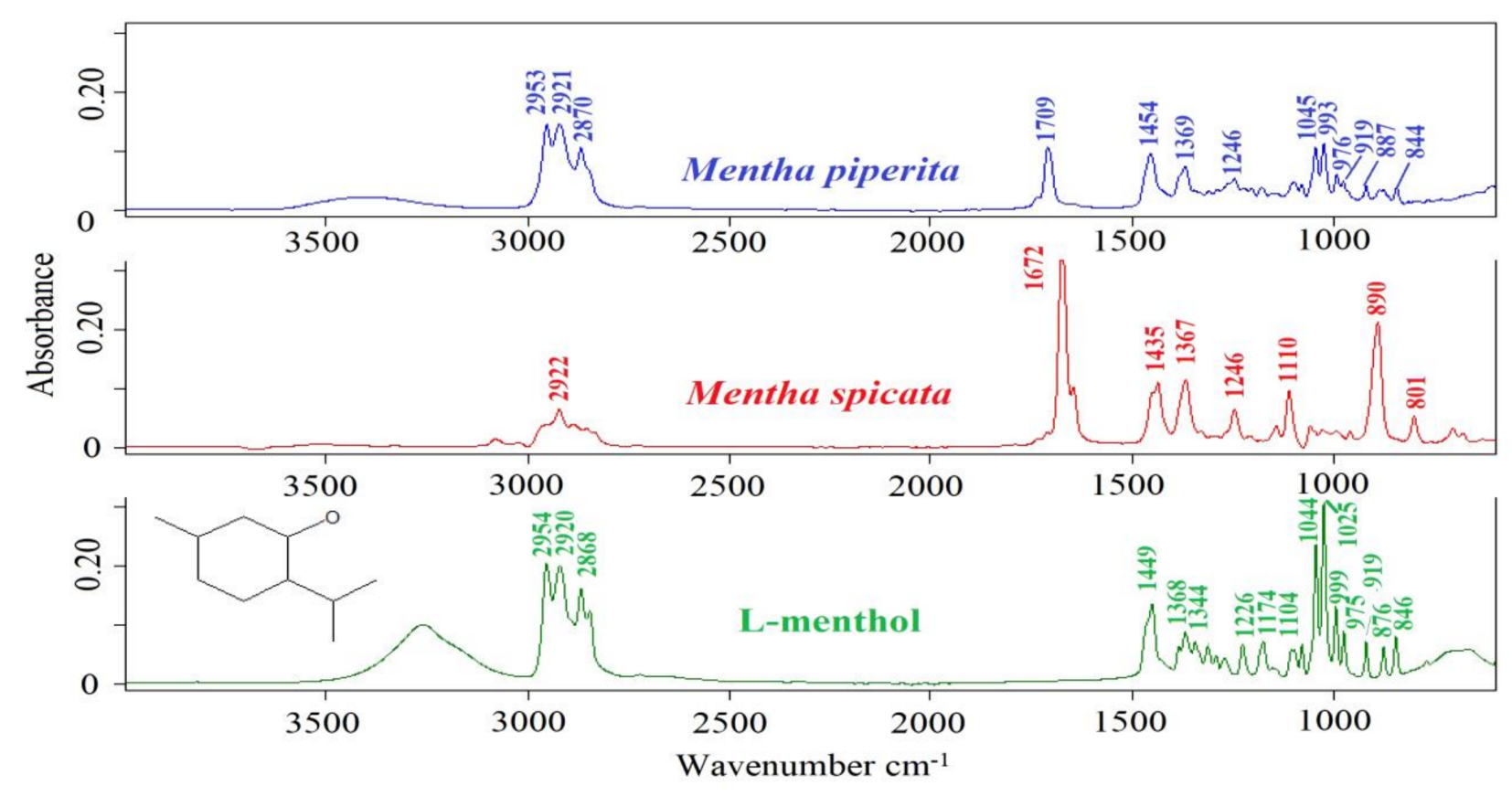

Figure 1. ATR-FTIR spectra of Mentha piperita EO, Mentha spicata EO and L-menthol in the $4000-400 \mathrm{~cm}^{-1}$ spectral region.

The band at $1709 \mathrm{~cm}^{-1}$ is due to the $-\mathrm{C}=\mathrm{O}$ stretching vibrations [19]. The vibrational band at $1454 \mathrm{~cm}^{-1}$ could be attributed to deformation vibrations of $\mathrm{CH}_{2}$ and $\mathrm{CH}_{3}$ groups [20]. The peak at $1369 \mathrm{~cm}^{-1}$ corresponds to the stretching vibrations of $-\mathrm{C}-\mathrm{H}$ and $-\mathrm{CH}_{3}$ groups [19]. The spectral band at $1246 \mathrm{~cm}^{-1}$ and $1045 \mathrm{~cm}^{-1}$ may be assigned to the $-\mathrm{C}-\mathrm{O}$ stretching vibrations or $-\mathrm{CH}_{2}$ - deformation vibrations and $-\mathrm{C}-\mathrm{O}$ stretching vibrations, respectively [19]. The peaks at $993 \mathrm{~cm}^{-1}$ and $887 \mathrm{~cm}^{-1}$ could be attributed to the $(-\mathrm{HC}=$ $\mathrm{CH}-$, trans-) bending vibrations. 
Table 1. Assignment of FTIR spectral bands for essential oils [13,16,18-20]. $v$-stretching vibrations, $\delta$-deformation vibrations, s-symmetric, as-asymmetric.

\begin{tabular}{|c|c|}
\hline IR $\left(\mathrm{cm}^{-1}\right)$ & Assignment \\
\hline 3470 & $\mathrm{OH}$ \\
\hline 3416 & $-\mathrm{C}=\mathrm{O}$ (overtone) \\
\hline $3100-3000$ & C-H (Alkene) \\
\hline $3150-3050$ & $\mathrm{C}-\mathrm{H}$ (aromatic) \\
\hline 2923,2875 & $v_{\text {as }}\left(-\mathrm{C}-\mathrm{H},-\mathrm{CH}_{2}\right)$ and $v_{\mathrm{s}}(-\mathrm{C}-\mathrm{H},-\mathrm{CH})$ \\
\hline 2950 & $v_{\mathrm{as}}\left(-\mathrm{C}-\mathrm{H},-\mathrm{CH}_{3},-\mathrm{CH}_{2}\right)$ \\
\hline 1708 & $v(-\mathrm{C}=\mathrm{O})$ in acid \\
\hline 1737 & $v(-\mathrm{C}=\mathrm{O})$ in ester \\
\hline 1708 & $v(-\mathrm{C}=\mathrm{O})$ in acid \\
\hline 1648 & $v(-\mathrm{C}=\mathrm{C}-$, cis- $)$ and $\delta(-\mathrm{OH})$ \\
\hline 1573 & Aromatic ring $\mathrm{C}=\mathrm{C}$ skeleton \\
\hline $1450 \mathrm{~cm}$ & $\mathrm{CH}_{2}$ deformation and asymmetrical $\mathrm{CH}_{3}$ deformation \\
\hline 1420 & $\mathrm{C}=\mathrm{CH}_{2}$ in-plane deformation vibration \\
\hline $1372 / 1337$ & $\left(-\mathrm{C}-\mathrm{H},-\mathrm{CH}_{3}\right)$, banding \\
\hline $1285 / 1244$ & $v(-\mathrm{C}-\mathrm{O})$ or $\delta\left(-\mathrm{CH}_{2}-\right)$ \\
\hline 1124 & C-O stretching \\
\hline 1116 & $v(-\mathrm{C}-\mathrm{O})$ or $\delta\left(-\mathrm{CH}_{2}-\right)$ \\
\hline 1094 & $v(-\mathrm{C}-\mathrm{O})$ \\
\hline $1044 / 1023$ & $v(-\mathrm{C}-\mathrm{O})$ \\
\hline $991 / 923$ & $\delta(-\mathrm{HC}=\mathrm{CH}-$, trans- $)$ bending out of plane \\
\hline 810 & C-H out-of-plane bending \\
\hline 805 & $\delta(-\mathrm{HC}=\mathrm{CH}-$, cis- $)$ bending out of plane $\delta\left(-\left(\mathrm{CH}_{2}\right) \mathrm{n}-\right.$ \\
\hline 770 & $\delta\left(-\left(\mathrm{CH}_{2}\right) \mathrm{n}-\right.$ and $-\mathrm{HC}=\mathrm{CH}-($ cis- $)$ bending (rocking) \\
\hline 685 & Alkenes \\
\hline
\end{tabular}

\subsection{Discrimination of Authentic Mentha piperita EOs from Adulterated Samples by HCA and} PCA

One of the aims of this study was to distinguish authentic Mentha piperita EO from spiked samples, Mentha spicata and L-menthol, with the help of discriminative techniques, such as hierarchical cluster analysis (HCA) and principal component analysis (PCA). HCA and PCA analysis was performed by using 1st derivatized FTIR spectra of all samples through Euclidian distance and Ward's algorithm. A spectral range of 4000 to $600 \mathrm{~cm}^{-1}$ was selected for HCA and PCA. Previous Data from several studies reported that the 4000-600 $\mathrm{cm}^{-1}$ spectral range included fingerprinting spectral information, which could be used to obtain classification patterns of authentic and counterfeit herbal samples [21]. HCA is an algorithmic approach and it provides an opportunity to observe the classification pattern of authentic and adulterated samples on 2-D dendrogram plots [22]. Dendrograms are composed of branches which include sample sets and sub-sets. Current research utilized Ward's algorithm to distinguish authentic Mentha piperita EOs from other samples. As basic principle, Ward's algorithm joins at each stage of the cluster pair whose merger minimizes the increase in the total within-group error sum of squares and Ward's algorithm is not restricted with the general classification problems [23]. Numerous studies employed Ward's algorithm to determine authentic samples because of the effectiveness of Ward's algorithm on the discriminative analyses [11,20,24-27]. It could be concluded from previous studies that the application of HCA coupled with Ward's algorithm reveals the hidden relationship between investigated samples on the basis of their FTIR spectra. HCA dendrogram of the current study is presented in Figure 2A. As can be seen, Mentha piperita samples were clearly distinguished from other Mentha species (Mentha spicata), L-menthol and adulterated samples. All samples were mainly classified as two main clusters. The right arm of the dendrogram (number 1) included Mentha spicata species and Mentha spicata adulterated samples in high concentration. The left arm of the dendrogram (number 2) included authentic Mentha piperita samples (marked with a green rectangle), 
L-menthol and Mentha piperita adulterates samples. Adulterated samples were marked by a red rectangle in Figure 2A. HCA not only distinguished the authentic Mentha piperita samples, but also presented a classification pattern that revealed the adulteration levels of spiked samples. In other words, the nearest subset to the authentic Mentha piperita samples included adulterated samples with $4 \%$ spiking level. Precise classification of all samples was obtained with a minimum adulteration level of $4 \%$ by using HCA and PCA. 3D-PCA results were presented in Figure 2B. We observed three well-separated clusters and Mentha piperita EOs were clearly distinguished from Mentha spicata, L-menthol, and adulterated samples. Results from HCA and PCA were coherent with each other. However, HCA provided information about classification patterns and the relationship between the elements of each set and sub-sets.

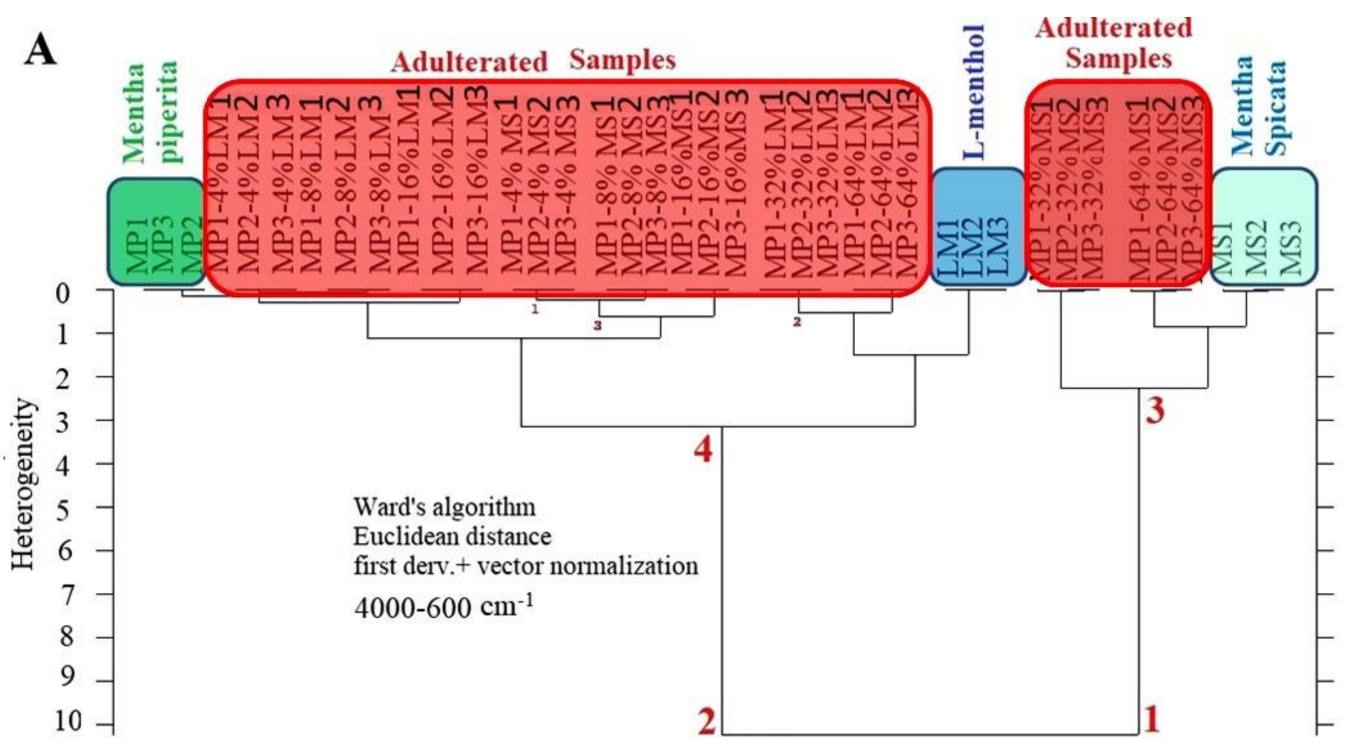

B

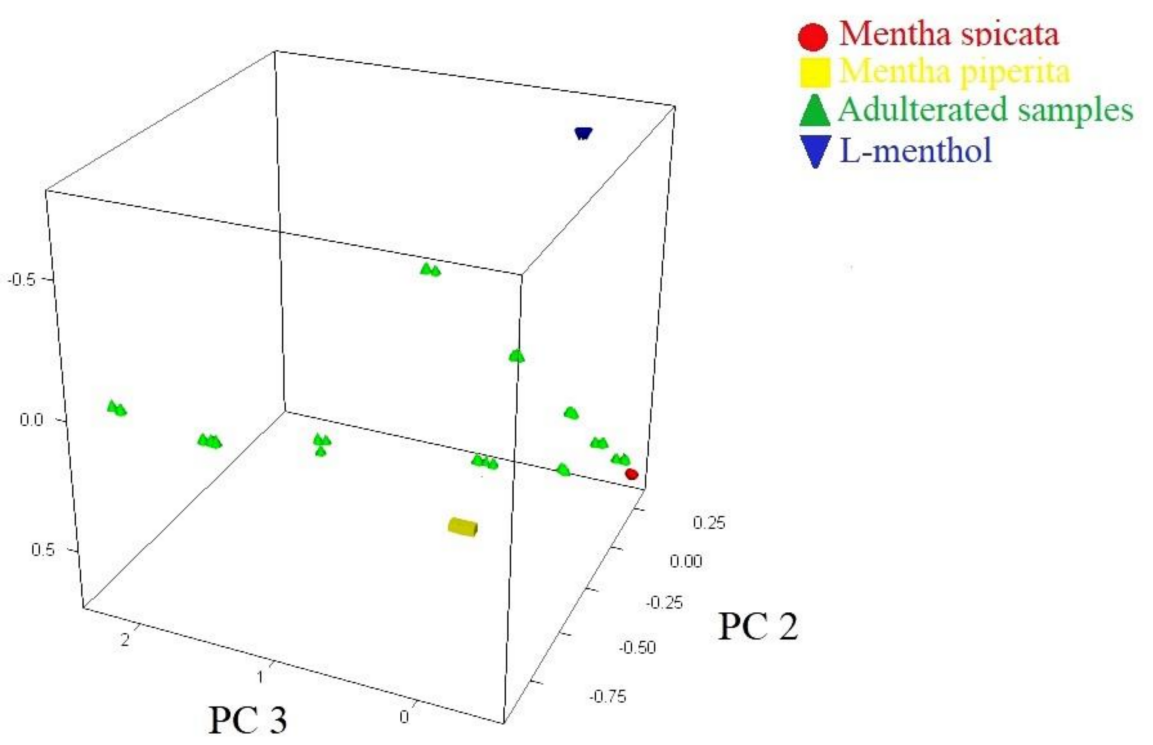

Figure 2. HCA dendrogram of FTIR spectra from Mentha piperita, Mentha spicata, L-menthol, and adulterated samples (A) 3-D PCA plot of FTIR spectra from Mentha piperita, Mentha spicata, L-menthol, and adulterated samples (B). 


\subsection{Prediction of Mentha spicata and L-Menthol Contents of Adulterated Mentha piperita Samples}

The current research used two different multivariate calibration models (PLSR and PCR) for the prediction of Mentha spicata and L-menthol contents of adulterated Menhta piperita samples. These powerful chemometrics are widely used to detect and quantify suspicious additives or adulterants in the various matrices such as foods, beverages, and essential oil for the sustainability of originality of high-valued products [22]. In the present study, research calibration and cross-validation was carried out for the samples of Mentha piperita EO (MP1, MP2, MP3), Mentha spicata EO (MS1, MS2, MS3), L-menthol (LM1, LM2, LM3) and adulterated Mentha piperita EOs at the concentration levels of 0, 1, 2, 4, 8, 16, 32, $64,100 \%(v / v)$. Calibration and cross-validation models were developed by using raw, 1st derivative and 2 nd derivative spectra of all sample set. These models were built by using specific spectral ranges for each adulterant. Previous contributions reported that the selected spectral range should involve spectral properties describing the concentration variation of the analyte or adulterant [28]. The spectral regions of 1066 to $1034 \mathrm{~cm}^{-1}$ and 1694 to $1651 \mathrm{~cm}^{-1}$ were chosen to build calibration and cross-validation models which have spectral information to predict L-menthol and Mentha piperita, respectively. These spectral regions were presented visually in Figure 3A,D. As can be seen, the absorbance intensity of the spectra increased proportionally with the rise in analyte quantity. Additionally, three dimensional spectra of adulterated samples $(0,1,2,4,8,16,32,64,100 \%(v / v))$ were illustrated in Figure 3C,F for adulterants of L-menthol and Mentha spicata. 3-D spectra visuals were plotted by using OriginPro software (OriginLab, Northampton, MA, USA). Cross-validation plots, regression equations, and regression coefficient $\left(R^{2}\right)$ are presented in Figure 3B,E for PLSR-raw spectra of L-menthol and Mentha spicata, respectively. The success of PLSR and PCR models were evaluated by the Press, Bias, and SECV values according to the previous reports [28]. " $\mathrm{R}^{2 "}$ is defined as the regression coefficient and it must be changed between " 0 " and " 1 ". When $R^{2}$ equals 1.0 , all points lie exactly on a straight line with no scatter. This means that $X$ values let one predict $Y$ values perfectly in the developed regression model [29]. Bias could be defined as the systematic error of the calibration or cross-validation and calculated as the average difference between the reference and predicted values. The standard error of cross-validation (SECV )is calculated as the square root of the residual variance divided by the number of degrees of freedom. The success (performance) of the models were evaluated using SECV (standard error of cross-validation).

$$
\mathrm{SECV}=\sqrt{\frac{\sum_{i=1}^{m}\left(c_{i}-c_{i}\right)^{2}}{m-2}}
$$

where $c_{i}$ is the reference and $\hat{c}_{i}$ is predicted concentration values of ith sample, $m$ is the number of samples. The degree of freedom is $m-2$ because when a linear model is assumed, there are only two parameters to be extracted, which are the slope of the actual vs. reference concentration plot and the intercept [30].

The results of bias, SECV, and press values are presented in Table 2. Favorable $\mathrm{R}^{2}$ values between 0.99 and 1 were obtained for both calibration and cross-validation models of all PLSR and PCR analyses. Then, three latent variables were selected to obtain minimum SECV values in all models. SECV values changed between 0.06 and 2.14. Additionally, bias and press values showed alteration between 0.06 to 1.43 and 0.03 to 41.15 , respectively. Results showed that PLSR and PCR models had the considerable capability to quantify Mentha spicata and L-menthol in Mentha piperita essential oil with high $\mathrm{R}^{2}$ values and low SECV and Bias values. 


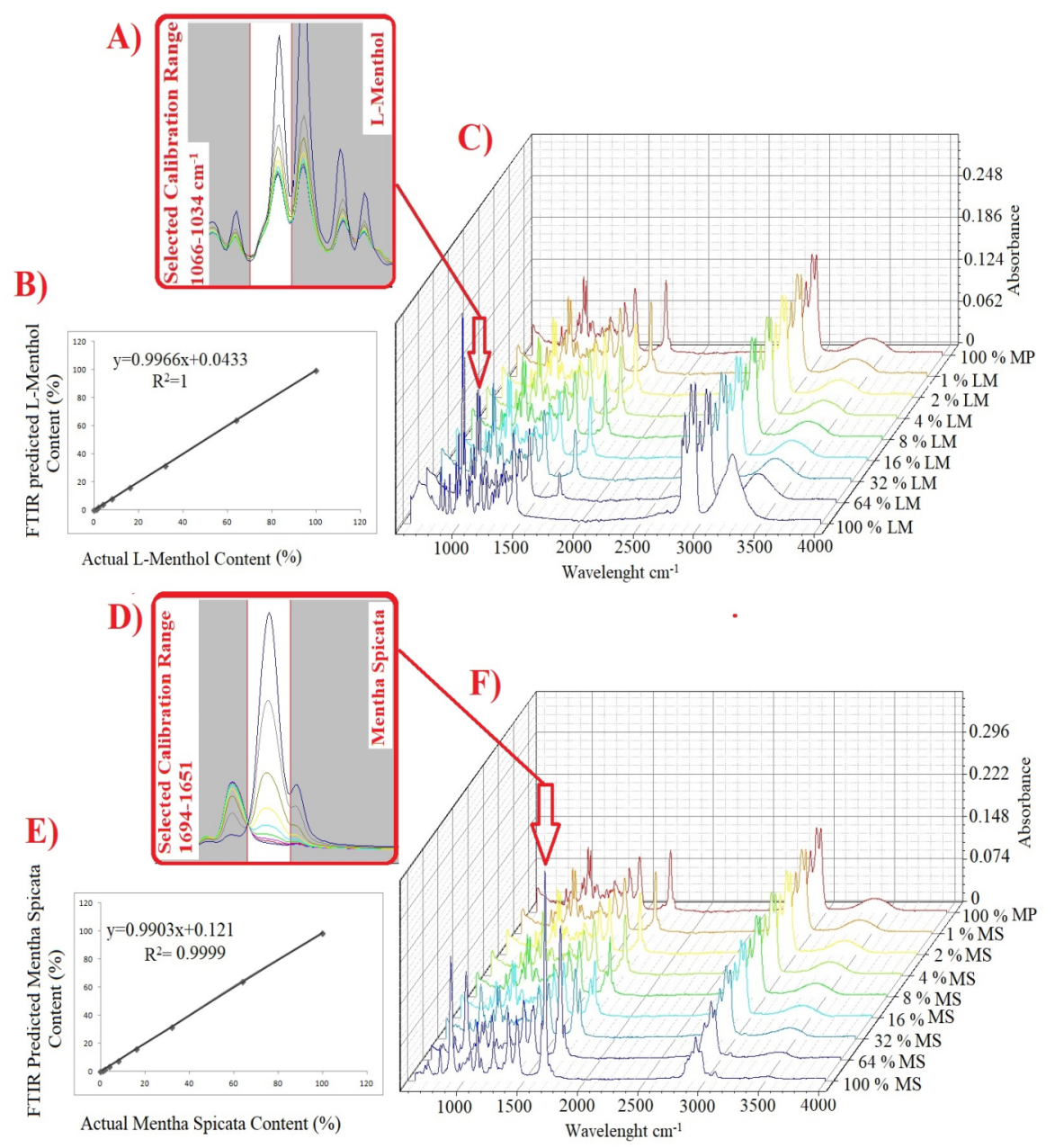

Figure 3. Selected spectral range for quantification L-menthol (A) PLSR-raw spectra regression cross-validation plot for Lmenthol (B) 3-D superimposed FTIR spectra of L-menthol adulterated samples (C) Selected spectral range for quantification Mentha spicata (D) PLSR-raw spectra regression cross-validation plot for Mentha spicata (E) 3-D superimposed FTIR spectra of Mentha spicata adulterated samples (F). 
Table 2. PLSR and PCR calibration and cross-validation results of raw, first and second derivative FTIR spectra of Mentha piperita essential oil.

\begin{tabular}{|c|c|c|c|c|c|c|c|c|c|}
\hline \multirow{2}{*}{ Sample Codes } & \multirow{2}{*}{ Model } & \multirow{2}{*}{ Preprocessing } & \multicolumn{2}{|c|}{ Equation } & \multicolumn{2}{|c|}{$\mathrm{R}^{2}$} & \multirow{2}{*}{ Press } & \multirow{2}{*}{ SECV } & \multirow{2}{*}{ Bias } \\
\hline & & & Calibration & Validation & Calibration & Validation & & & \\
\hline \multirow{5}{*}{$\begin{array}{c}\text { MS1 MP1 } \\
\text { (MS1 adulterated } \\
\text { MP1) }\end{array}$} & \multirow{2}{*}{ PLSR } & Raw & $y=1 x+1 \times 10^{-4}$ & $y=0.9903 x+0.121$ & $\mathrm{R}^{2}=1$ & $\mathrm{R}^{2}=0.9999$ & 2.15 & 0.49 & 0.47 \\
\hline & & Second derivative. & $y=1 x+0.0001$ & $y=0.9942 x+0.0198$ & $\mathrm{R}^{2}=1$ & $\mathrm{R}^{2}=0.9999$ & 1.83 & 0.45 & 0.21 \\
\hline & \multirow{3}{*}{ PCR } & Raw & $y=1 x+1 \times 10^{-4}$ & $y=0.9918 x+0.1041$ & $\mathrm{R}^{2}=1$ & $\mathrm{R}^{2}=0.9999$ & 1.61 & 0.42 & 0.40 \\
\hline & & First derivative. & $y=1 x+0.0001$ & $y=1.0068 x-0.0767$ & $\mathrm{R}^{2}=1$ & $\mathrm{R}^{2}=0.9999$ & 1.03 & 0.34 & 0.32 \\
\hline & & Second derivative. & $y=1 x+0.0001$ & $y=0.9939 x+0.022$ & $\mathrm{R}^{2}=1$ & $\mathrm{R}^{2}=0.9999$ & 1.36 & 0.39 & 0.22 \\
\hline \multirow{5}{*}{$\begin{array}{c}\text { MS2 MP2 } \\
\text { (MS2 adulterated } \\
\text { MP2) }\end{array}$} & \multirow{3}{*}{ PLSR } & Raw & $y=1 x+5 \times 10^{-5}$ & $y=0.9977 x+0.0314$ & $\mathrm{R}^{2}=1$ & $\mathrm{R}^{2}=1$ & 0.16 & 0.13 & 0.11 \\
\hline & & First derivative. & $y=1 x+0.0002$ & $y=0.9914 x+0.0821$ & $\mathrm{R}^{2}=1$ & $\mathrm{R}^{2}=0.9994$ & 5.10 & 0.75 & 0.45 \\
\hline & & Second derivative. & $y=1 x+0.0003$ & $y=0.9905 x+0.0618$ & $R^{2}=1$ & $\mathrm{R}^{2}=0.9998$ & 10.05 & 1.06 & 0.35 \\
\hline & \multirow[b]{2}{*}{ PCR } & Raw & $y=1 x+4 \times 10^{-5}$ & $y=0.9988 x+0.0178$ & $\mathrm{R}^{2}=1$ & $\mathrm{R}^{2}=1$ & 0.07 & 0.09 & 0.06 \\
\hline & & First derivative. & $y=1 x+0.0002$ & $y=0.9906 x+0.0901$ & $R^{2}=1$ & $\mathrm{R}^{2}=0.9993$ & 5.24 & 0.76 & 0.48 \\
\hline \multirow{6}{*}{$\begin{array}{c}\text { MS3 MP3 } \\
\text { (MS3 adulterated } \\
\text { MP3) }\end{array}$} & \multirow{3}{*}{ PLSR } & Raw & $y=1 x+4 \times 10^{-5}$ & $y=0.9966 x+0.0433$ & $\mathrm{R}^{2}=1$ & $\mathrm{R}^{2}=1$ & 0.29 & 0.18 & 0.16 \\
\hline & & First derivative. & $y=1 x+4 \times 10^{-5}$ & $y=1.0006 x-0.0019$ & $\mathrm{R}^{2}=1$ & $\mathrm{R}^{2}=1$ & 0.03 & 0.06 & 0.04 \\
\hline & & Second derivative. & $y=1 x+8 \times 10^{-5}$ & $y=0.9892 x+0.0713$ & $\mathrm{R}^{2}=1$ & $R^{2}=0.9998$ & 4.82 & 0.73 & 0.41 \\
\hline & \multirow{3}{*}{ PCR } & Raw & $y=1 x+4 \times 10^{-5}$ & $y=0.9983 x+0.0247$ & $\mathrm{R}^{2}=1$ & $\mathrm{R}^{2}=1$ & 0.12 & 0.11 & 0.09 \\
\hline & & First derivative. & $y=1 x+4 \times 10^{-5}$ & $y=1.0006 x-0.0024$ & $\mathrm{R}^{2}=1$ & $\mathrm{R}^{2}=1$ & 0.03 & 0.06 & 0.04 \\
\hline & & Second derivative. & $y=1 x+8 \times 10^{-5}$ & $y=0.9885 x+0.0764$ & $\mathrm{R}^{2}=1$ & $R^{2}=0.9998$ & 4.83 & 0.73 & 0.43 \\
\hline
\end{tabular}


Table 2. Cont.

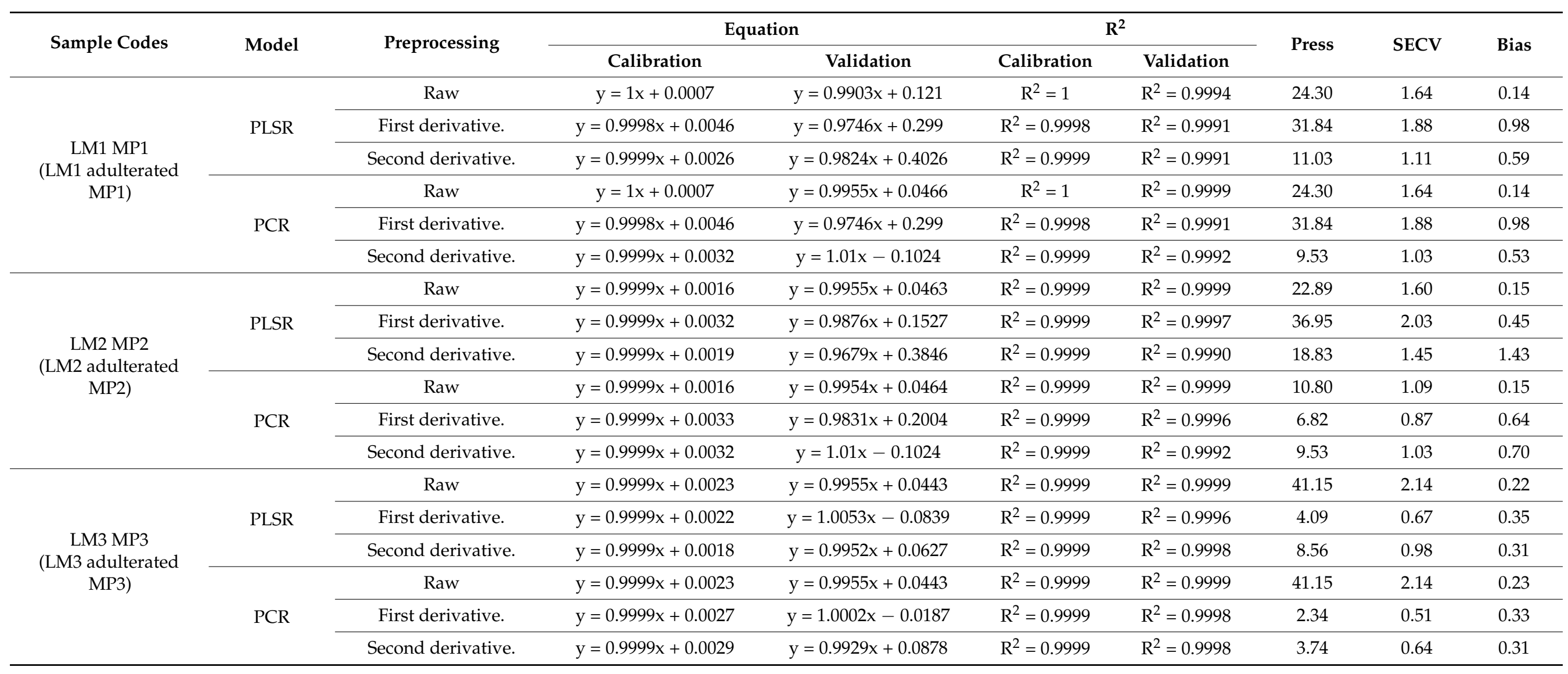




\section{Discussion}

The current study evaluated the capability of rapid, non-destructive, reliable and robust ATR-FTIR spectroscopy coupled with multivariate analyses for detection and quantification of adulterants in Mentha piperita EOs. Quality evaluation of Mentha piperita EOs is performed using various analysis according to the international standard [3]. Mainly chromatographic analyses are performed to observe the chemical composition of essential oils based on international standard. Chromatographic methods are reliable and accurate, but they are generally laborious, time-consuming, high-cost and more complicated when compared to ATR-FTIR spectroscopy [31]. Results from earlier studies demonstrated the success of ATR-FTIR spectroscopy combined with chemometrics for the determination of the authenticity of essential oils. A previous study determined the geographical origin of Grosso lavandin essential oils of controlled area (GLEOCA) using ATR-FTIR spectroscopy combined with chemometrics and the geographic origin was successfully evaluated using PCA plots [12]. In another study, researchers utilized vibrational spectroscopy with the aid of chemometrics for characterization of high-value geranium oil as an alternative to conventional gas chromatography technique and the content of marker compounds were determined using PLSR multivariate calibration models [15]. In the previous studies, multivariate statistics of PCR and PLSR were successfully employed to calculate main essential oil components such as carvacrol, linalool, myrcene, thymol, etc. Additionally, hierarchical cluster analyses were successfully employed to classify various chamomile oils obtained from different chemotypes and manufacturing processes [32]. Recent evidence suggests that ATR-FTIR spectroscopy can be used as a green, direct, reliable, robust, and cost-effective analytical technique for quality control of essentials oils [20].

To the best of our knowledge, current research is the first attempt for detection and quantification of adulterants of Mentha spicata EO and L-menthol in Mentha piperita EO using ATR-FTIR spectroscopy coupled with multivariate analyses of PLSR, PCR, HCA, and PCA. Discriminative methods of HCA and PCA were successfully applied to distinguish authentic Mentha piperita EO from fraudulent samples on the basis of FTIR spectra. Previous researches mostly dealt with the characterization and antimicrobial properties of Mentha piperita EO [4,16,19,20,33-35]. Other researches included gas chromatography-mass spectrometry(GC-MS) and PCR (polymerase chain reaction) analysis of essential oils from Mentha species [34,36]. Only the studies of Sadowska et al. (2019) and Agatonovic-Kustrin et al. (2020) used FTIR spectroscopy to characterize Mentha piperita species and successfully performed band assignments of FTIR spectra from Mentha piperita EO. Our FTIR characterization results for Mentha piperita $E O$ were quite similar to those they found. Our results showed that HCA and PCA could effectively distinguish authentic Mentha piperita EOs from fraudulent samples. In accordance with the present results, previous studies have demonstrated that HCA and PCA can be effectively used for the determination of adulterants in various oil species such as olive oil and mustard oil using ATR-FTIR spectra $[37,38]$.

Additionally, the current study showed that ATR-FTIR spectroscopy coupled with PLSR and PCR had the capability to quantify adulterants (Mentha spicata and l-menthol) in Mentha piperita EO. These results seem to be consistent with other researches which quantified various adulterants in oil matrices such as hazelnut oil, olive oil, and extra virgin olive oil using vibrational spectroscopy coupled with PLS models [39-41].

\section{Conclusions}

The main goal of the current study was to determine the concentrations of Mentha spicata EOs and L-menthol in the adulterated Mentha piperita EOs using ATR-FTIR spectroscopy coupled with PLSR and PCR. The second aim of this study was to distinguish authentic Mentha piperita EOs from adulterated samples, Mentha spicata EOs and L-menthol samples.

Adulterant contents (Mentha spicata and L-menthol) were successfully calculated using PLSR and PCR models at the concentration range of 0 to $100 \%(v / v)$. SECV values changed 
between 0.06 and 2.14. Additionally, bias and press values showed alteration between 0.06 and 1.43 and 0.03 and 41.15, respectively. PLSR and PCR showed high accuracy by using raw, 1st derivative and 2nd derivative spectra of all samples. In other words, quite favorable prediction results were obtained utilizing developed all PLSR and PCR models. Additionally, the discriminative analysis was performed to distinguish authentic Mentha piperita essential oil from adulterated samples, Mentha spicata and L-menthol. A clear classification pattern was observed on 2-D dendrograms. Authentic Mentha piperita EOs distinctly classified from adulterated samples and adulterants using cluster analyses of HCA and PCA. These results showed that ATR-FTIR spectroscopy coupled with HCA and PCA could be effectively employed in discrimination of different Mentha species, as well as foreign synthetic chemicals, diluents, and low-quality essential oils. The current study performed characterization of Mentha piperita EO, Mentha spicata EO, and L-menthol by using ATR-FTIR spectroscopy, thus fingerprinting ATR-FTIR data of these samples were contributed to the scientific knowledge.

Taking into account all the mentioned results, FTIR spectroscopy can be considered an appropriate, new, effective, reliable, low-cost and green analytical technique for quality control of essential oils such as Mentha piperita EO. Application of the developed models by portable or hand-held options in the whole supply chain may prevent trading of fraudulent essential oil samples.

Author Contributions: Conceptualization, O.S. and N.C.; Methodology, N.C.; Software, N.C.; Validation, N.C., O.T. and O.S.; Investigation, N.C.; Resources, N.C., O.T. and O.S.; Data Curation, N.C.; Writing-Original Draft Preparation, N.C. and O.S.; Writing-Review and Editing, N.C., O.T. and O.S.; Visualization, N.C.; Supervision, N.C.; Project Administration, N.C., O.T. and O.S. All authors have read and agreed to the published version of the manuscript.

Funding: This project was funded by the Deanship of Scientific Research (DSR) at King Abdulaziz University, Jeddah, under grant no. (FP-58-42). The authors, therefore, acknowledge with thanks to DSR technical and financial support.

Conflicts of Interest: The authors declare that they have no known competing financial interests or personal relationships that could have appeared to influence the work reported in this paper.

\section{References}

1. Trevisan, S.C.C.; Menezes, A.P.P.; Barbalho, S.M.; Guiguer, É.L. Properties of Mentha piperita: A brief review. World J. Pharm. Med. Res. 2017, 3, 309-313.

2. Mahendran, G.; Rahman, L.U. Ethnomedicinal, phytochemical and pharmacological updates on Peppermint (Mentha piperita L.)-A review. Phyther. Res. 2020, 34, 2088-2139. [CrossRef] [PubMed]

3. International Standard ISO 856:2006. Oil of Peppermint. (Mentha piperita L.); ISO: Geneva, Switzerland, 2006. Available online: https:/ / www.iso.org/standard/32041.html (accessed on 10 December 2020).

4. Ruiz Del Castillo, M.L.; Blanch, G.P.; Herraiz, M. Natural variability of the enantiomeric composition of bioactive chiral terpenes in Mentha piperita. J. Chromatogr. A 2004, 1054, 87-93. [CrossRef]

5. Anwar, F.; Abbas, A.; Mehmood, T.; Gilani, A.H.; Rehman, N. Mentha: A genus rich in vital nutra-pharmaceuticals-A review. Phyther. Res. 2019, 33, 2548-2570. [CrossRef] [PubMed]

6. Johnson, R. Food fraud and "Economically motivated adulteration" of food and food ingredients. In Food Fraud and Adulterated Ingredients: Background, Issues, and Federal Action; Congressional Research Service: Washington, DC, USA, 2014 ; pp. 1-56.

7. Burfield, T. The Adulteration of Essential Oils and the Consequences to Aromatherapy \& Natural Perfumery Practice. In Proceedings of the International Federation of Aromatherapists Annual AGM, London, UK, 11 October 2003.

8. NIIR Board. The Complete Technology Book of Essential Oils (Aromatic Chemicals); National Institute of Industrial Research: New Delhi, India, 2003.

9. Bounaas, K.; Bouzidi, N.; Daghbouche, Y.; Garrigues, S.; de la Guardia, M.; El Hattab, M. Essential oil counterfeit identification through middle infrared spectroscopy. Microchem. J. 2018, 139, 347-356. [CrossRef]

10. Rohman, A.; Arsanti, L.; Erwanto, Y.; Pranoto, Y. The use of vibrational spectroscopy and chemometrics in the analysis of pig derivatives for halal authentication. Int. Food Res. J. 2016, 23, 1839-1848.

11. Baranska, M.; Schulz, H.; Walter, A.; Rösch, P.; Quilitzsch, R.; Lösing, G.; Popp, J. Investigation of eucalyptus essential oil by using vibrational spectroscopy methods. Vib. Spectrosc. 2006, 42, 341-345. [CrossRef] 
12. Bombarda, I.; Dupuy, N.; Da, J.P.L.V.; Gaydou, E.M. Comparative chemometric analyses of geographic origins and compositions of lavandin var. Grosso essential oils by mid infrared spectroscopy and gas chromatography. Anal. Chim. Acta 2008, 613, 31-39. [CrossRef]

13. Li, S.; Zhu, X.; Zhang, J.; Li, G.; Su, D.; Shan, Y. Authentication of pure camellia oil by using near infrared spectroscopy and pattern recognition techniques. J. Food Sci. 2012, 77, 374-380. [CrossRef]

14. Tankeu, S.Y.; Vermaak, I.; Kamatou, G.P.P.; Viljoen, A.M. Vibrational spectroscopy and chemometric modeling: An economical and robust quality control method for lavender oil. Ind. Crops Prod. 2014, 59, 234-240. [CrossRef]

15. Sandasi, M.; Kamatou, G.P.P.; Gavaghan, C.; Baranska, M.; Viljoen, A.M. A quality control method for geranium oil based on vibrational spectroscopy and chemometric data analysis. Vib. Spectrosc. 2011, 57, 242-247. [CrossRef]

16. Yilmaztekin, M.; Lević, S.; Kalušević, A.; Cam, M.; Bugarski, B.; Rakić, V.; Pavlović, V.; Nedović, V. Characterisation of peppermint (Mentha piperita L.) essential oil encapsulates. J. Microencapsul. 2019, 36, 109-119. [CrossRef] [PubMed]

17. Rohman, A.; Man, Y.B.C. Application of Fourier transform infrared spectroscopy for authentication of functional food oils. Appl. Spectrosc. Rev. 2012, 47, 1-13. [CrossRef]

18. Boughendjioua, H. Fourier transformed infrared spectroscopy analysis of constituents of lemon essential oils from algeria. Am. J. Opt. Photonics 2017, 5, 30. [CrossRef]

19. Sadowska, U.; Matwijczuk, A.; Dródz, T.; Zabinski, A.; Niemczynowicz, A. Spectroscopic Examination and chemometric analysis of essential oils obtained from Peppermint. Processes 2019, 7, 466. [CrossRef]

20. Agatonovic-Kustrin, S.; Ristivojevic, P.; Gegechkori, V.; Litvinova, T.M.; Morton, W.D. Essential oil quality and purity evaluation via ft-ir spectroscopy and pattern recognition techniques. Appl. Sci. 2020, 10, 7294. [CrossRef]

21. Singh, S.K.; Jha, S.K.; Chaudhary, A.; Yadava, R.D.S.; Rai, S.B. Quality control of herbal medicines by using spectroscopic techniques and multivariate statistical analysis. Pharm. Biol. 2010, 48, 134-141. [CrossRef]

22. Taylan, O.; Cebi, N.; Yilmaz, M.T.; Sagdic, O.; Ozdemir, D.; Balubaid, M. Rapid detection of green-pea adulteration in pistachio nuts using Raman spectroscopy and chemometrics. J. Sci. Food Agric. 2020. [CrossRef]

23. Murtagh, F.; Legendre, P. Ward's hierarchical clustering method: Clustering criterion and agglomerative algorithm. J. Classif. 2011, 31, 274-295. [CrossRef]

24. Cebi, N.; Yilmaz, M.T.; Sagdic, O. A rapid ATR-FTIR spectroscopic method for detection of sibutramine adulteration in tea and coffee based on hierarchical cluster and principal component analyses. Food Chem. 2017, 229, 517-526. [CrossRef]

25. Ropodi, A.I.; Pavlidis, D.E.; Mohareb, F.; Panagou, E.Z.; Nychas, G.J.E. Multispectral image analysis approach to detect adulteration of beef and pork in raw meats. Food Res. Int. 2015, 67, 12-18. [CrossRef]

26. Been, F.; Roggo, Y.; Degardin, K.; Esseiva, P.; Margot, P. Profiling of counterfeit medicines by vibrational spectroscopy. Forensic Sci. Int. 2011, 211, 83-100. [CrossRef] [PubMed]

27. Karami, A.; Zandi, P.; Khosh-khui, M.; Salehi, H.; Saharkhiz, M.J. Analysis of essential oil from nine distinct genotypes of Iranian Damask rose (Rosa damascena Mill). J. Med. Plants Res. 2012, 6, 5495-5498.

28. Sivakesava, S.; Irudayaraj, J. Detection of inverted beet sugar adulteration of honey by FTIR spectroscopy. J. Sci. Food Agric. 2001, 81, 683-690. [CrossRef]

29. Cebi, N.; Yilmaz, M.; Sagdic, O.; Yuce, H.; Yelboga, E. Prediction of peroxide value in omega-3 rich microalgae oil by ATR-FTIR spectroscopy combined with chemometrics. Food Chem. 2017, 225, 188-196. [CrossRef]

30. Başar, B.; Özdemir, D. Determination of honey adulteration with beet sugar and corn syrup using infrared spectroscopy and genetic-algorithm-based multivariate calibration. J. Sci. Food Agric. 2018, 98, 5616-5624. [CrossRef]

31. Ozen, B.F.; Weiss, I.; Mauer, L.J. Dietary supplement oil classification and detection of adulteration using Fourier transform infrared spectroscopy. J. Agric. Food Chem. 2003, 51, 5871-5876. [CrossRef]

32. Schulz, H.; Quilitzsch, R.; Krüger, H. Rapid evaluation and quantitative analysis of thyme, origano and chamomile essential oils by ATR-IR and NIR spectroscopy. J. Mol. Struct. 2003, 661-662, 299-306. [CrossRef]

33. Pramila, D.M. Phytochemical analysis and antimicrobial potential of methanolic leaf extract of peppermint (Mentha piperita: Lamiaceae). J. Med. Plants Res. 2012, 6, 331-335. [CrossRef]

34. Buleandra, M.; Oprea, E.; Popa, D.E.; David, I.G.; Moldovan, Z.; Mihai, I.; Badea, I.A. Comparative chemical analysis of Mentha piperita and M. spicata and a fast assessment of commercial peppermint teas. Nat. Prod. Commun. 2016, 11, 551-555. [CrossRef]

35. Tyagi, A.K.; Malik, A. Antimicrobial potential and chemical composition of Mentha piperita oil in liquid and vapour phase against food spoiling microorganisms. Food Control 2011, 22, 1707-1714. [CrossRef]

36. Capuzzo, A.; Maffei, M.E. Molecular fingerprinting of peppermint (Mentha piperita) and some Mentha hybrids by sequencing and RFLP analysis of the 5S rRNA Non-Transcribed Spacer (NTS) region. Plant Biosyst. 2016, 150, 236-243. [CrossRef]

37. Jamwal, R.; Amit; Kumari, S.; Balan, B.; Kelly, S.; Cannavan, A.; Singh, D.K. Rapid and non-destructive approach for the detection of fried mustard oil adulteration in pure mustard oil via ATR-FTIR spectroscopy-chemometrics. Spectrochim. Acta Part A Mol. Biomol. Spectrosc. 2021, 244, 118822. [CrossRef] [PubMed]

38. De la Mata, P.; Dominguez-Vidal, A.; Bosque-Sendra, J.M.; Ruiz-Medina, A.; Cuadros-Rodríguez, L.; Ayora-Cañada, M.J. Olive oil assessment in edible oil blends by means of ATR-FTIR and chemometrics. Food Control 2012, 23, 449-455. [CrossRef]

39. Öztürk, B.; Yalçin, A.; Özdemir, D. Determination of olive oil adulteration with vegetable oils by near infrared spectroscopy coupled with multivariate calibration. J. Near Infrared Spectrosc. 2010, 18, 191-201. [CrossRef] 
40. Pan, M.; Sun, S.; Zhou, Q.; Chen, J. A Simple and portable screening method for adulterated olive oils using the hand-held ftir spectrometer and chemometrics tools. J. Food Sci. 2018, 83, 1605-1612. [CrossRef]

41. Ozen, B.F.; Mauer, L.J. Detection of hazelnut oil adulteration using FT-IR spectroscopy. J. Agric. Food Chem. 2002, 50, 3898-3901. [CrossRef] 